\title{
Increase in root nodulation and crop yield of soybean by native Bradyrhizobium japonicum strains
}

\author{
Namraj Dhami ${ }^{*}$ and Braj Nandan Prasad ${ }^{2}$ \\ ${ }^{1}$ The School of Pharmaceutical and Biomedical Sciences, Pokhara University, Pokhara, Nepal; ${ }^{2}$ Central Department of \\ Botany, Tribhuvan University, Kirtipur, Kathmandu, Nepal; *Corresponding author, e-mail: nrdhami@gmail.com
}

\begin{abstract}
Native strains of Bradyrhizobium japonicum were tested for their effectiveness on nodulation, crop yield and nitrogen fixation in soybean (Glycine max). B. japonicum strains were isolated from soybean root nodules collected from different agro-climatic regions of Far Western Nepal, viz. Dipayal (607 m asl), Dadeldhura (1097 m asl), Silgadhi (1209 m asl) and Bajura (1524 m asl). The strains were characterized by studying colony characteristics, growth response with Congo red and Bromothymol blue, and Gram staining. The native bradyrhizobial strains were authenticated by performing infection test on soybean seedlings. All the four strains were found compatible and effective on root nodulation, crop yield and soil nitrogen $(\mathrm{N})$ content. Inoculation of these strains increased soybean root nodulation by $247-343 \%$ and crop yield by $45-204 \%$. There was strong positive correlation $(r=0.982)$ between number of root nodules and crop yield, which suggest that optimization of root nodulation by inoculating compatible and effective B. japonicum strains significantly increase the soybean crop yield. Soil $\mathrm{N}$ content of inoculated experimental pots was increased by $13-33 \%$. However, variability among different strains was observed in their effect on root nodulation and yield performance. B. japonicum strain collected from Silgadhi was found to be the most effective in increasing nodule number and crop yield by $343 \%$ and $204 \%$ respectively.
\end{abstract}

Key-words: Glycine max, gram staining, inoculation, nitrogen fixation.

\section{Introduction}

Soybean [Glycine max (L.) Merr.] is highly nutritional protein supplement and is used in the form of cooking oil or snacks items, as well as in the form of soya sauce, soya milk, etc. The protein content in soybean has been found up to $43 \%$ of its dry weight. Soybean production has increased by 10 folds (from 17 million tones in 1950 to 179 million tones in 2002) in the last 50 years. Soybean is one of the important legume crops in Nepal, where it is grown covering about 22,073 ha area with annual production of 19,362 tones (FAO 2004). The yield potential of soybean in mid hills of Nepal is $4.5 \mathrm{t} / \mathrm{ha}$ (NARC 2002), while average national yield is only $0.877 \mathrm{t} / \mathrm{ha}$ (FAO 2004).

One of the important factors affecting soybean production is the effectiveness of native bradyrhizobia existing in the soil. The bradyrhizobia present in all types of soil may not always be sufficient and efficient for providing adequate nitrogen to the growing soybean under existing conditions. Generally, the soil under continuous soybean cultivation is found to have higher bradyrhizobial populations, whereas the soil with little or no past history of soybean cultivation does not contain sufficient bradyhizobial populations and even if present most of the bradyrhizobia from soybean-free soil have been found to be nonsymbiotic (Pongslip et al. 2002). The symbiotic efficiency and competitiveness of bradyrhizobia determine the yield performance of soybean. In Nepalese perspective, lack of effective bradyrhizobial population is the main obstacle for soybean cultivation (Pant and Prasad 2004). Thus, it is imperative to screen the most efficient and competent bradyrhizobia for inoculation purpose for soybean cultivation. In the present work, the effect of native bradyrhizobial strains on root nodulation and crop yield of soybean and nitrogen (N) content of soil was studied.

\section{Materials and Methods}

ISOLATION OF BRADYRHIZOBIUM JAPONICUM ISOLATES

Native B. japonicum strains were isolated from effective soybean root nodules collected from four different agro-climatic regions of far-western Nepal representing different altitudinal ranges, viz. Dipayal (DPL, 607 m asl), Dadeldhura (DDL, $1097 \mathrm{~m}$ asl), Silgadhi (SIL, $1209 \mathrm{~m}$ asl) and Bajura (BJR, $1524 \mathrm{~m}$ asl). Bradyrhizobial strains were cultured, isolated and purified by using Yeast Extract Mannitol Agar(YMA) medium (Vincent 1970), and Congo red (Somasegaran and Hoben 1994). Purified cultures were later stored at $4 \pm 1^{\circ} \mathrm{C}$ in YMA slants.

\section{CHARACTERIZATION AND AUTHENTICATION OF THE ISOLATES}

The native bradyrhizobial isolates were characterized by studying their presumptive characteristics, such as colony characteristics, culture response on Congo red, Bromothymol blue and Gram staining. Furthermore, the isolates were authenticated by performing infection test on sterile sand using Jensen's N-free medium and Yeast Extract Mannitol Broth (YMB) of bradyrhizobial isolates (Somasegaran and Hoben 1994; Arroyo et al. 1998). The inoculated and uninoculated soybean seedlings were examined for nodulation after three weeks. 


\section{POT EXPERIMENT}

Pots of $22 \mathrm{~cm}$ height and $24 \mathrm{~cm}$ diameter were swabbed with absolute alcohol before 24 hours and filled with 2:1 garden soil and sterilized sand. Surface sterilized seeds of Sathiya cultivar of soybean (Glycine max) were sown in the pots. YMB $(15 \mathrm{ml})$ of different native bradyrhizobial isolates with $4 \times 107$ CFU (colony forming units) was inoculated in pots (one isolate in one set of experimental pots), maintaining four replicates for each set and one set was kept uninoculated. After one week, soybean seedlings were thinned and only four healthy seedlings were maintained in each pot. Adequate water was provided to the growing plants until harvesting. Number of nodules and nodule dry matter content of inoculated and uninoculated soybean plants were recorded on 60th day after seed sowing. The yield parameters, such as number of pods, seed biomass (weight of 100 seeds) and total yield of soybean were also recorded. The nitrogen content of soil samples collected before soybean sowing and after soybean harvesting was estimated by Kjeldahl method (Bergersen 1980).

\section{Results}

Native bradyrhizobial strains were successfully isolated from effective soybean root nodules collected from different sites of farwestern Nepal. Translucent creamy and raised bradyrhizobial colonies of 1.5 to $2 \mathrm{~mm}$ diameter were obtained after 7 days of culture in YMA plates. The bacterial colonies did not absorb any red color of Congo red in dark culture that facilitated the isolation and purification of bradyrhizobia. Furthermore, we observed alkali production during culture and gram negative responses of the isolates. The isolates were confirmed to be the Bradyrhizobium japonicum strains as they all produced effective nodules on soybean seedlings grown on sterilized sand. Thus, isolates, DPL, DDL, SIL and BJR, were authenticated as B. japonicum strains.

Inoculation of all four native $B$. japonicum strains increased root nodulation and crop yield (Tables 1 and 2). Inoculation increased the yield two to three times over uninoculated plants. We found significant positive correlations between nodule number and crop yield ( $r=0.982, p=0.01$; Table 3 ). In inoculated plants, the number of nodules increased by 247 to $343 \%$ (Table 2). Nodule dry matter and other parameters (number of pods and seed biomass) were also increased in inoculated plants (Tables 1 and 2).

The soil N content was increased up to $33.37 \%$ after soybean harvesting (Table 4). The amount of $\mathrm{N}$ addition to the soil was correlated with the number of nodules $(p<0.05)$. The experimental sets in which plants were with higher number of nodules were found to have more $\mathrm{N}$ in the soil.

Although all the four strains were found to be efficient for root nodulation and all of them significantly increased the crop yield, there was strong variation in the degree of effect on these parameters among the four strains. B. japonicum strain 'SIL' was found to be the most efficient strain (Tables 1 and 2).
Table 1. Effect of Bradyrhizobium japonicum strains on nodulation and growth parameters in soybean after 60 days of sowing.

\begin{tabular}{lccccc}
\hline $\begin{array}{l}\text { B. japonicum } \\
\text { strains }\end{array}$ & NN/pl. & $\begin{array}{c}\text { NDM/pl. } \\
(\mathrm{g})\end{array}$ & POD/pl. & $\begin{array}{c}100 \mathrm{~s} \\
(\mathrm{~g})\end{array}$ & $\begin{array}{c}\text { Yield/pl. } \\
(\mathrm{g})\end{array}$ \\
\hline Control & 35.75 & 0.19 & 32.37 & 17.36 & 6.67 \\
DPL & 124.25 & 0.34 & 45.62 & 19.94 & 14.02 \\
DDL & 133.25 & 0.36 & 43.50 & 21.49 & 16.38 \\
SIL & 158.50 & 0.49 & 45.25 & 25.08 & 20.27 \\
BJR & 137.75 & 0.41 & 43.62 & 22.33 & 17.20 \\
\hline
\end{tabular}

Strains: DPL-Dipayal, DDL-Dadeldhura, SIL-Silgadhi, BJR-Bajura. Growth and yield parameters: NN/pl.-nodule number per plant, NDM/ pl.-nodule dry matter per plant, POD/pl.-number of pods per plant, 100s-weight of 100 seeds.

Table 2. Percent increase in nodulation and growth parameters in bradyrhizobium-inoculated soybean over uninoculated plants.

\begin{tabular}{lccccc}
\hline \multirow{2}{*}{$\begin{array}{l}\text { B. japonicum } \\
\text { strains }\end{array}$} & \multicolumn{5}{c}{ Percent increase in } \\
\cline { 2 - 6 } & NN/pl. & NDM/pl. & POD/pl. & 100s & Yield/pl. \\
\hline DPL & 247.55 & 78.94 & 40.93 & 14.86 & 110.19 \\
DDL & 272.72 & 89.47 & 34.58 & 23.79 & 145.57 \\
SIL & 343.35 & 157.89 & 39.78 & 44.47 & 203.89 \\
BJR & 285.31 & 115.78 & 34.75 & 28.62 & 157.87 \\
\hline
\end{tabular}

Abbreviations as in Table 1.

Table 3. Relationships between nodulation and soybean growth and yield parameters.

\begin{tabular}{lccc}
\hline & NN & NDM & POD \\
\hline NDM & $0.957^{*}$ & & \\
POD & $0.955^{*}$ & $0.852^{*}$ & \\
Yield & $0.982^{* *}$ & $0.987^{* *}$ & $0.882^{*}$ \\
\hline *Correlation is significant at the 0.05 level (2 tailed); **Correlation is
\end{tabular}
significant at the 0.01 level ( 2 tailed).

NN-nodule number, NDM-nodule dry matter, POD-number of pod.

Table 4. Nitrogen (N) addition to the soil by native Bradyrhizobium japonicum strains.

\begin{tabular}{llcc}
\hline SN & Soil samples & N content of soil (\%) & Increase in N content (\%) \\
\hline 1 & sbss & 0.060 & \\
2 & sash CTL & 0.067 & 11.67 \\
3 & sash DPL & 0.068 & 13.34 \\
4 & sash DDL & 0.071 & 18.34 \\
5 & sash SIL & $0.080^{\star}$ & $33.37^{\star}$ \\
6 & sash BJR & 0.073 & 21.67 \\
\hline
\end{tabular}

sbss $=$ soil before soybean sowing, sash $=$ soil after soybean harvesting, *shows the best performance

\section{Discussion}

Bradyrhizobial strains were isolated from soybean root nodules. The longer duration of colony development showed slow growing nature of bradyrhizobia. Somasegaran and Hoben (1994) also suggested the requirement of 7-10 days incubation for bradyrhizobia. Alkali production during culture and gram negative responses supported the characterization of bradyrhizobial isolates.

Inoculation of all four native B. japonicum strains increased 
root nodulation and crop yield. High degree of correlation between nodule number and crop yield (Table 3) suggested that the optimization of nodulation play crucial role in increasing soybean yield. Due to the lack of sufficient B. japonicum cells in the soil, plants were having less root nodules in uninoculated sets, whereas in inoculated plants, the number of nodules increased by 247 to $343 \%$ over uninoculated plants. Nodule dry matter also increased with the increase in nodule number. Larger bradyrhizobial population infected more root hairs enhancing the nodule number, ultimately contributing to the higher dry matter of nodules per plant. Several studies also reported significant increase in soybean growth parameters and yield due to the inoculation of bradyrhizobial isolates (e.g., Purcino et al. 2000; Okereke et al. 2001; Pant and Prasad 2004). Okereke et al. (2001) observed 71 to $486 \%$ increment in nodule number due to the inoculation of B. japonicum isolates on soybean, which is comparable with the present finding.

The plants having higher number of nodules were found to possess high nodule dry matter and better crop yield than uninoculated plants. It indicates that the degree of root nodulation determines the crop yield in soybean. Sato et al. (1999) suggested that optimizing the nodulation could maximize the nitrogen fixation. According to Nelson et al. (1984), rate of nitrogen fixation in root nodules determines the overall performance of soybean growth, development and yield. The soil $\mathrm{N}$ content was increased up to $33.37 \%$ after soybean harvesting. The amount of $\mathrm{N}$ addition to the soil was correlated with the number of nodules. The increase in soil $\mathrm{N}$ content after soybean harvesting is thus due to degradation of senescent root nodules.

The results of this study suggest that inoculation of effective B. japonicum strain invariably increases root nodulation in soybean that in turn increases crop yield. The differences in the nodulation and crop yield parameters reveal the variability in the efficiency of native B. japonicum strains. Such variability in effectiveness of different rhizobial strains was also reported from other regions (e.g., Arroyo et al. 1998; Minamisawa et al. 1999; Nuntagij 1999). In the present study, the B. japonicum strain 'SIL' was found to be the most efficient strain in producing such effects. It is emphasized that nitorgen fixing efficiency may vary among different strains of B. japonicum. Therefore, inoculation of compatible and effective $B$. japonicum strain is necessary to optimize the soybean root nodulation that significantly increases the crop yield and improves the soil $\mathrm{N}$ status. The addition of $\mathrm{N}$ increases soil fertility that in turn plays an important role for subsequent crops. Thus, the use of effective bradyrhizobial isolates as biofertilizer could be the highly sustainable practice for soybean cultivation as well as to reduce the increasing demand of chemical fertilizers.

\section{Acknowledgements}

Authors are thankful to the Biotechnology Unit, Central Department of Botany, Tribhuvan University, Kathmandu for laboratory facilities and cooperation during the research work.

\section{References}

Arroyo J.V., Sessitsch A., Martinez E. and Pena-Cabriales J.J. 1998. Nitrogen fixation and nodule occupancy by native strains of Rhizobium on different cultivars of common bean (Phaseolus vulgaris L.). Plant and Soil 204: 147-154.

Bergersen F.J. 1980. Methods for Evaluating Biological Nitrogen Fixation. John Wiley and Sons Ltd., New York, USA.

FAO 2004. Food and Agriculture Organization Yearbook. www.faostat.fao.org.

Minamisawa K., Nakatsuka Y. and Isawa T. 1999. Diversity and field site variation of indigenous populations of soybean bradyrhizobia in Japan by fingerprints with repeated sequences RSa and RSb. FEMS Microbiol Ecology 29: 171-178.

NARC 2002. Technical Annual Report. Agronomy Division, Nepal Agricultural Research Council, Kathmandu, Nepal.

Nelsol D.R., Bellville R.J., Zampini C.A. and Maxwell C.A. 1984. Interactions between carbon and nitrogen during pod filling. In: Proceedings of World Soybean Research Conference III August 12-17, 1984 (R. Shibles, ed.), pp. 824-832. Westview Press, London, UK.

Nuntagij A., Wadisirisuk P., Kotepong S., Panuvas V. and Boonkerd N. 1999. Selection of $B$. japonicum strains for effectively fixed $\mathrm{N}_{2}$ under acid soil condition. In: Evaluation of Nitrogen Fixing Bacteria in South Asia (Y. Murooka, ed.), pp. 263-272. Osaka University, Osaka, Japan.

Okereke G.U., Onochie G., Onukwo A. and Onyeagba E. 2001. Effectiveness of foreign bradyrhizobia strains in enhancing nodulation, dry matter and seed yield of soybean [G. $\max$ (L.) Merr.] cultivars in Nigeria. Biology and Fertility of Soils 33: 3-9.

Pant B.D. and Prasad B.N. 2004. Effectiveness of Bradyrhizobium isolates on seedling growth and nitrogen content in soybean [Glycine max (L.) Merr.]. Botanica Orientalis 4: 1-3.

Pongslip N., Teaumroong N., Nuntagij A., Boonkerd N. and Sadowsky M.J. 2002. Genetic structure of indigeneous non-nodulating and nodulating populations of Bradyrhizobium in soils from Thailand. Symbiosis 33: 39-58.

Purcino H.M.A., Festin P.M. and Elkan G.H. 2000. Identification of effective strains of Bradyrhizobium for Arachis pintoi. Tropical Agriculture 77: 226-231.

Sato T., Yashima H., Ontake N., Sueyoshi K., Akao S. and Ohyama T. 1999. Possible involvement of photosynthetic supply in changes of nodule characteristics of hypernodulating soybeans. Soil Science and Plant Nutrition 45: 187-196.

Somasegaran P. and Hoben J. 1994. Handbook for Rhizobia: Methods in Legume-Rhizobium Technology. Springer-Verlag, New York, USA.

Vincent J.M. 1970. A Manual for the Practical Study of the Root Nodule Bacteria. IBP Handbook No. 15. International Biological Programme, Blackwell Scientific Publications, Oxford, UK. 